\title{
1 Single-cell classification using learned cell phenotypes
}

2 Yang Chen ${ }^{1}$, Tadepally Lakshmikanth ${ }^{1}$, Jaromir Mikes ${ }^{1}$, Petter Brodin ${ }^{1,2}$

$4 \quad{ }^{1}$ Science for Life Laboratory, Department of Women's and Children's Health,

5 Karolinska Institutet, Sweden

${ }^{2}$ Pediatric Rheumatology, Karolinska University Hospital, Sweden

Correspondence: petter.brodin@ki.se

\section{Abstract}

12 Single-cell methods such as flow cytometry, Mass cytometry and single-cell mRNA sequencing collect high-dimensional data on thousands to millions of individual cells. An important aim during the analysis of such data is to classify cells into known categories and cell types. One commonly used approach towards this is clustering of cells with similar features followed by manual annotation of clusters in relation to known biology. A second approach, commonly used for cytometry data relies on manual sorting or "gating" of cells, often based on pairwise combinations of measurements used in a stepwise and very tedious process of cell annotation. Both of these approaches require manual inspection and annotation of every new dataset generated, a process that is not

21 only time consuming but also subjective and surely influential for the conclusions drawn.

22 The manual annotation is also difficult to reproduce by other researchers with a different

23 perception of features that signify their cells of interest. Here we propose an alternative

24 strategy based on machine learning of known phenotypes from manually curated, high-

25 dimensional data and thereby enabling rapid classification of subsequent datasets in a more reproducible manner. This simple approach increases both throughput, reproducibility and simplicity of cell classification in single-cell biology. 
32 The number of methods for generating high-dimensional single-cell data is rapidly

33 expanding. These methods are enabling multi-scale analyses from thousands to

34 millions of cells, resulting in unprecedented levels of details in our analyses of

35 heterogeneous tissues (Davis and Brodin, 2018a). (Davis and Brodin, 2018b). The most

36 widely used single-cell technology to date has been flow cytometry, developed more

37 than 50 years ago, and still a workhorse in the fields of immunology, cancer biology and

38 cell biology. Flow cytometry allows for the rapid enumeration of close to 30 parameters

39 in millions of cells using antibodies coupled to fluorescent probes. With the application

40 of mass cytometry, the number of measured parameters in any given cell has increased

41 to > 50 (Brodin, 2019). Single-cell genomic methods is also advancing rapidly and now

42 allow for the quantification of thousands of mRNA-molecules, epigenetic states, or DNA-

43 sequence variants, all in tens of thousands of cells (Macaulay et al., 2017). After

44 normalization and various other steps of pre-processing, one important task in the

45 analysis of such high-dimensional single-cell data, is to classify cells into defined populations that can be interrogated and interpreted in relation to what is already known with respect to known biological functions. In flow and mass cytometry, this process is most commonly done by manually inspecting individual markers and pairwise combinations of markers with known distribution, through an iterative process of "gating", cells are manually classified into the many known populations of cells. For blood immune cell about 20 different cell lineages and many subpopulations have been described (Uhlen et al., 2019). This process is intractable when the number of measured features grows above a certain level, such as in mass cytometry and singlecell mRNA-sequencing datasets (Mair et al., 2016). This manual gating procedure must also be repeated in full, with every new sample, and every experiment. Given these well-known limitations, several methods for automated cell classification have been

57 introduced in recent years (Mair et al., 2016). There is now a plethora of tools for 58 clustering of single-cell data, and the specific tool used in the field largely depends on

59 the type of data, and the habit of the investigator although some systematic

60 comparisons have been performed (Weber and Robinson, 2016). One common feature 61 of existing tools today, is the requirement for clusters of cells, like manually gated cell 62 populations, to be curated by the investigator in analyzing each new dataset. This 
63 represents another laborious and subjective process, that strongly influences the 64 downstream conclusions. The subjective nature of this cluster annotation also makes it 65 difficult to document, and therefor also difficult to reproduce, by other investigators.

66 Even if complete misclassification of cells is probably uncommon in these instances,

67 subtle variations in the interpretation of cell phenotypes will occur that make comparisons across studies difficult. To tackle these challenges, we propose an alternative strategy, in which cell classification is manually performed, based on expert knowledge, only during the assembly of a reference dataset, and this dataset is then used to train a classifier algorithm that will be able to classify similar cells rapidly and with good accuracy in additional datasets generated under comparable conditions. This approach increases the speed at which cell classification can occur, but also makes the process more reproducible.

We use large scale mass cytometry datasets to illustrate our approach, although we anticipate this strategy will be equally useful for other single-cell datasets. We first built a software for gating training datasets, not based on traditional biaxial plots as is commonly done during flow cytometry "gating". Instead we embedded a dataset using Barnes-Hut-SNE (Maaten, 2013) and gated training data based on all relevant antigens in the panel. In the first step, we embed an entire whole blood dataset of $\sim 1,000,000$ cells using bhSNE and color the data by canonical lineage-defining markers such as

83 CD3, CD4, CD8, CD14, CD56 and CD19, until all subpopulations have been identified

84 and manually gated in the bh-SNE embedded data. Larger clusters, such as CD4 ${ }^{+}$Tcells, CD8 ${ }^{+}$T-cells, B-cells, NK-cells, and Monocytes are subsequently embedded separately in a second level bh-SNE embedding, taking only features relevant for the given cell type into account. The purpose of this second layer of selection is to identify and label subpopulations of cells of importance (Maecker et al., 2012), and include

89 these when training the learning algorithm (Figure 1). Examples of such subpopulations identified in this second level classification are memory and naïve lymphocyte subsets,

91 and canonical subpopulations of monocytes and NK-cells (Figure 1A). It is important to

92 note that these subpopulations can be more difficult to discriminate at the first level 
94 labeled training data, such as manual flow cytometry gating and supervised or

95 unsupervised clustering of cells can also be used for the following training algorithm.

97 After the training dataset of high quality has been classified and single-cell tables have 98 been appended with cell labels, we are ready to train a classifier algorithm. The training 99 process employs 4 common machine learning algorithms; Logistic regression (Yu et al., 100 2011), Random forest (Ho, 1998), support-vector machine (SVM)(Cortes and Vapnik, 101 1995), Extreme gradient boosting (XGBoost)(Friedman, 2001) Figure 1b). For each of 102 these algorithms, the F1-score is calculated from precision and recall tests against an 103 additional manually classified dataset not included during the training procedure (Figure 104 1c). From the mean and standard deviation of the F1-score, the best performing model 105 is selected and used henceforth.

107 Particles - Level 0

\begin{tabular}{|l|l|}
\hline Population & F1 score \\
\hline Cells & 0.99901 \\
\hline Non-cells & 0.93633 \\
\hline
\end{tabular}

108

109 Whole blood - Level 1

\begin{tabular}{|l|l|}
\hline Population & F1 score \\
\hline B & 0.99576 \\
\hline Basophils & 0.99509 \\
\hline CD4T & 0.99458 \\
\hline CD8T & 0.98649 \\
\hline Eosinophils & 0.99377 \\
\hline gdT & 0.97842 \\
\hline MAIT & 0.92922 \\
\hline Monocytes & 0.98639 \\
\hline Neutrophils & 0.99740 \\
\hline NK & 0.98161 \\
\hline pDC & 0.98293 \\
\hline
\end{tabular}

111 CD4 $^{+}$T-cells - Level 2

\begin{tabular}{|l|l|}
\hline Population & F1 score \\
\hline Central memory CD4T & 0.82282 \\
\hline Effector memory CD4T & 0.85544 \\
\hline Memory Tregs & 0.83341 \\
\hline
\end{tabular}




\begin{tabular}{|l|l|}
\hline Naïve CD4T & 0.98413 \\
\hline Naïve Tregs & 0.92499 \\
\hline TEMRA CD4T & 0.55489 \\
\hline
\end{tabular}

113 B-cells - Level 2

\begin{tabular}{|l|l|}
\hline Population & F1 score \\
\hline $\operatorname{lgD}^{+}$Memory B & 0.74591 \\
\hline IgD- memory B & 0.88233 \\
\hline Naive B & 0.98359 \\
\hline Plasmablasts & 0.06452 \\
\hline Transitional B & 0.48740 \\
\hline
\end{tabular}

115 NK-cells - Level 2

116

\begin{tabular}{|l|l|}
\hline Population & F1 score \\
\hline CD56bright NK & 0.95993 \\
\hline CD56dim NK & 0.97871 \\
\hline
\end{tabular}

117 CD8 ${ }^{+}$T-cells - Level 2

\begin{tabular}{|l|l|}
\hline Population & F1 score \\
\hline Activated CD8 T & 0.70737 \\
\hline Central memory CD8 T & 0.73160 \\
\hline DP T-cells & 0.90411 \\
\hline Effector memory CD8T & 0.81967 \\
\hline Naive CD8 T & 0.98115 \\
\hline
\end{tabular}

119 Monocytes - Level 2

120

\begin{tabular}{|l|l|}
\hline Population & F1 score \\
\hline Classical Mono & 0.98874 \\
\hline Non-classical Mono & 0.83288 \\
\hline Proinflammatory mono & 0.93532 \\
\hline
\end{tabular}

$121 \gamma \delta$ T-cells - Level 2

\begin{tabular}{|l|l|}
\hline Population & F1 score \\
\hline CD161+gdT & 0.98340 \\
\hline CD161- gdT & 0.95848 \\
\hline
\end{tabular}

122 
a Defining training data

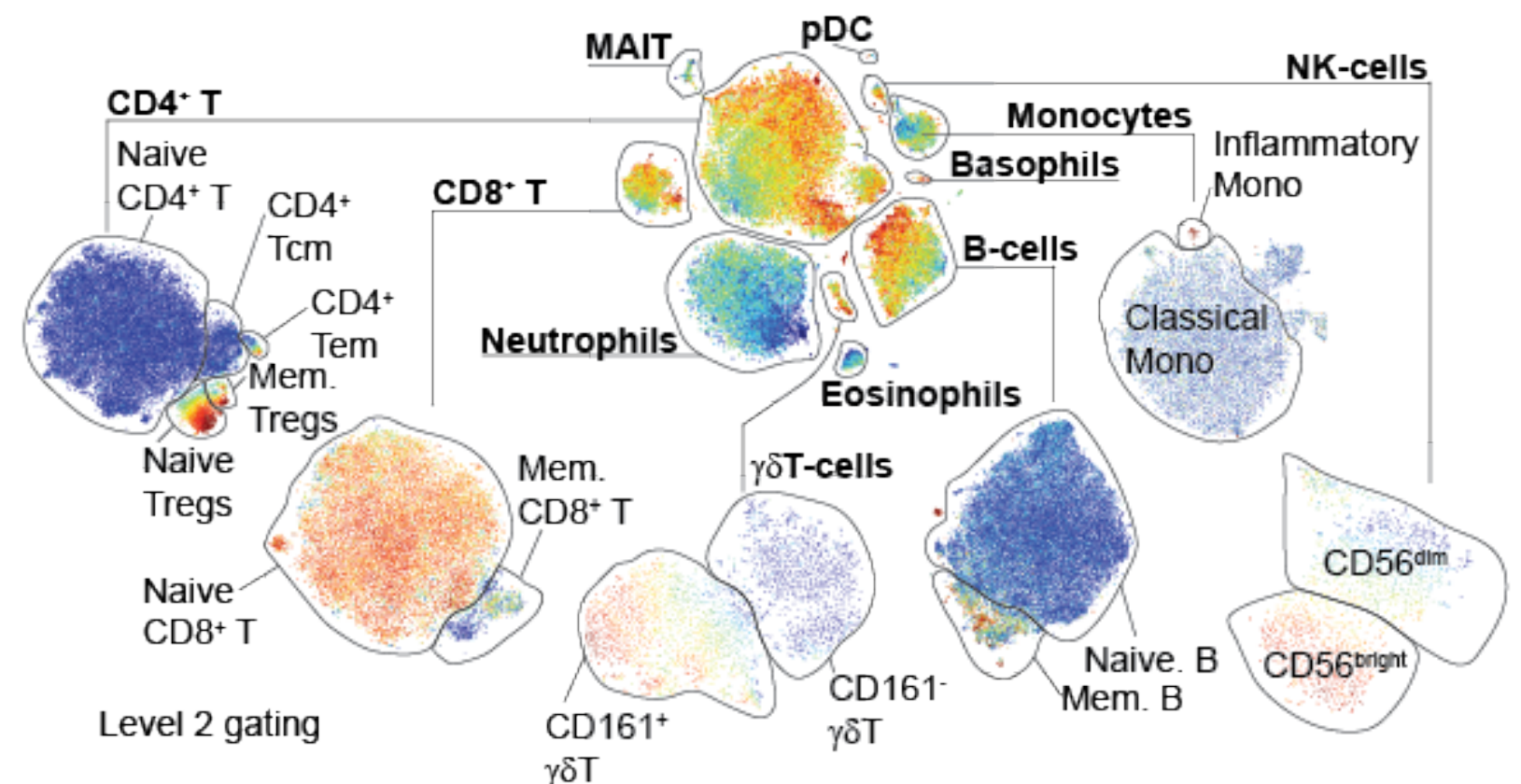

b Train multiple models

$$
\begin{aligned}
& \text { Logistic regression } \\
& \text { Random forest } \\
& \text { SVM } \\
& \text { XGBoost }
\end{aligned}
$$

Artificial Neural Network

\section{d Model comparison (F1-score)}

\section{C}

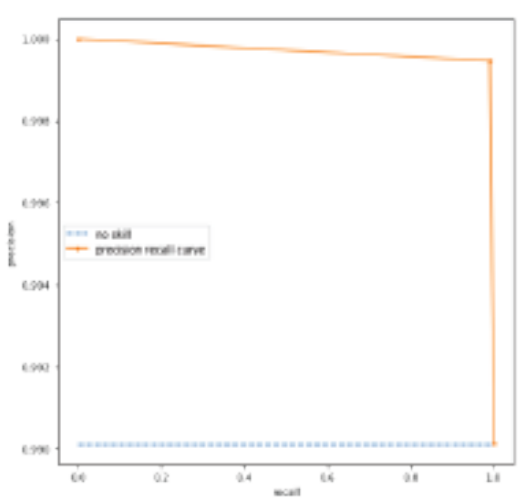

\begin{tabular}{|l|l|l|l|l|l|}
\hline & $\begin{array}{l}\text { Logistic } \\
\text { regression }\end{array}$ & $\begin{array}{l}\text { Random } \\
\text { forest }\end{array}$ & SVM & XGBoost & ANN \\
\hline F1 mean & 0.99799 & 0.99918 & 0.99771 & 0.99929 & $0.999 B$ \\
\hline F1 std & 0.00022 & 0.00013 & 0.00016 & 0.00010 & 0.00011 \\
\hline
\end{tabular}

Figure 1. a) Manual gating using a hierarchical tSNE embedded mass cytometry data. The indicated example markers are highlighted in color from blue (low) to red (high). b) The classified training data is used to train different learning algorithms. c) Precision/recall curves. d) F1-score comparison of trained models.

129 The classification of subsequently generated datasets can now be performed in a fully 130 automated fashion. Classifier finishes 1,000,000 cells in 36 seconds, and we recently 131 classified a large experiment with 400 large FCS-files in 48 minutes while manual gating of comparable 34 subsets would take weeks to finish. It is important to note that the 
133 performance of the classifier must be investigated by visualization of the data output to

134 ensure correct cell labeling. This is particularly important when test data is different from

135 the training data as this obviously increases the uncertainty in the classification. We

136 therefore redo training with the inclusion of new training data on a regular basis and

137 redo the learning process.

Another important aspect of this approach is the ability to assess feature importance for 140 the classification accuracy. This analysis can help guide better panel design in flow and 141 mass cytometry in a data-driven manner to maximize the information content in a panel.

142 This is done by iteratively applying the XGBoost classifier on the manually classified test 143 dataset. In each loop one marker is excluded and the accuracy of the classification is 144 monitored. From loss of precision, a score of importance can be assigned to each 145 feature in the dataset (Figure 2a). The results of this exercise are shown for level 1 146 classification of 11 canonical blood immune cell populations and reveals that the most 147 important marker is CD38, followed by CD99. We noticed that lineage markers such as 148 CD19, CD20, CD20 and CD8a were absent from the list of the top 10 most important 149 markers. This illustrates the ability of a data-driven approach such as this machine 150 learning approach to guide better panel design in mass cytometry and flow cytometry. 151 Similarly, we assess marker importance at each of the level 2 learning steps and report 152 these in Figure 2c. We conclude from these findings that a machine learning approach 153 weighting 4 common classification algorithms, represents an efficient method for 154 automating cell classification by considering manually classified training data. The 155 approach is fully reproducible, and the steps of cell classification are traceable when 156 applied to novel data. The method also represents a massive increase in throughput for 157 mass cytometry data analysis over manual gating, with hundreds of files readily 158 classified in only a few seconds. 


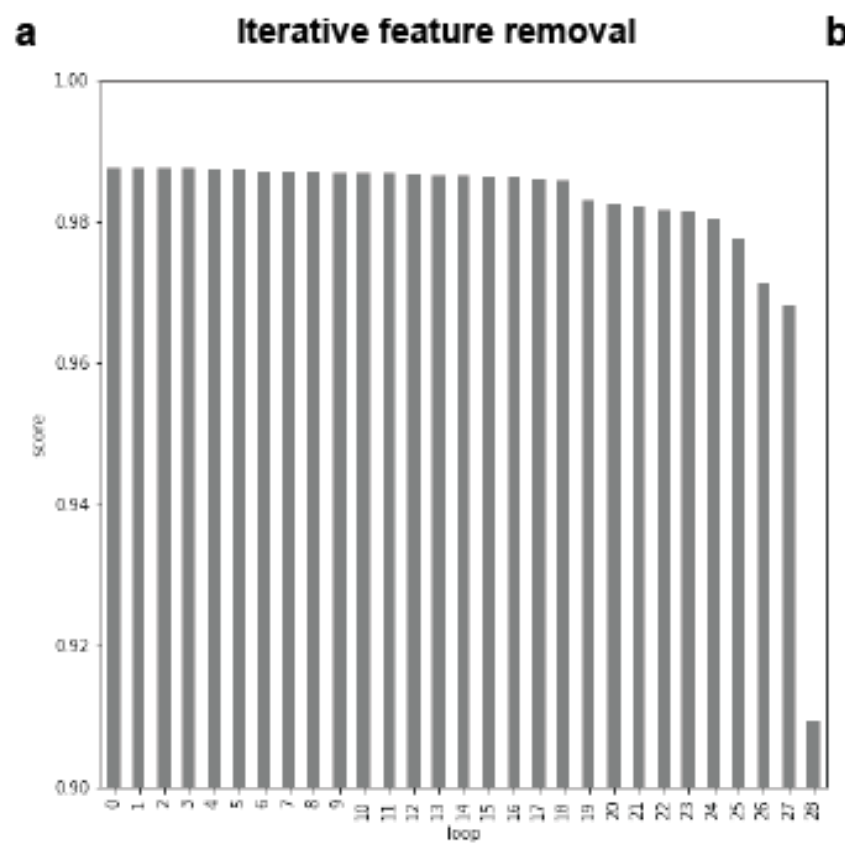

b Level 1 classificlefion

\begin{tabular}{|l|l|}
\hline & importance \\
\hline marker & \\
\hline CD4 & 0.081571 \\
\hline CD7 & 0.082208 \\
\hline CD24 & 0.083544 \\
\hline CD44 & 0.086963 \\
\hline CD3e & 0.088672 \\
\hline CD11b & 0.089153 \\
\hline CD45 & 0.089977 \\
\hline CD45RA & 0.091919 \\
\hline HLA-DR & 0.093504 \\
\hline CD99 & 0.103743 \\
\hline CD38 & 0.108746 \\
\hline & \multicolumn{2}{|c}{171} \\
\hline
\end{tabular}

172 Figure 2. a) Iterative leave-one-marker out test of model performance reveal relative importance of each marker in the mass cytometry antibody panel. b) at level 1 cell populations classification top 11 markers are indicated for the example dataset classified in figure 1.

176 There is a need to improve both the throughput and reproducibility of classification of

177 cell populations from single-cell data. Such datasets are now very commonly used but

178 the conclusions drawn about biological processes, differences in cell composition and

179 function in relation to health and disease, all depend upon the annotation of individual

180 cells into canonical or novel cell populations. Manual processes have dominated in the

181 past, particularly when flow cytometry datasets are used. Manual gating has several

182 important caveats. It is intractable for datasets with hundreds of samples, millions of

183 cells and tens to hundreds of features measured per cell. Also, manual annotation is

184 subjective and difficult to reproduce and thereby influence the conclusions drawn from

185 the data. The benefit of the manual and supervised analyses methods are that prior

186 knowledge allow for rapid quality control of the data, there is a large amount of human

187 experience involved in looking at the data which allow for biological interpretation and

188 hypothesis formulation, not possible by any algorithms. 
190 Here we present a very simple, yet powerful method for combining the interpretability of

191 manual annotation, with the scalability, throughput, speed and reproducibility that

192 automated methods allow. By using a manually annotated training dataset and learning

193 cell phenotypes associated with annotations such as immune cell subsets, we are able

194 to rapidly classify and annotate new datasets generated and still be able to draw

195 biological conclusions from the data and relate these immediately to existing body of

196 literature. A second important feature of this approach is that markers used for

197 classification can be ranked by importance and thereby allow for optimization of

198 antibody panels or mRNA-probes or other predefined panels of features analyzed. We

199 provide the learning and classifier algorithms as a standalone software package for

200 others to use freely. This software takes a defined input format and learns indicated cell

201 population labels for future classification tasks. The main limitation of this work is that it

202 is intrinsically difficult to develop a learning algorithm that performs equally well on all

203 populations of interest. We typically find that small subsets require more training data

204 and that subsets of cells without clear separation are obviously harder. In some

205 instances, we find it useful to classify the well separated subsets and then within such

206 subsets where phenotypes vary along a continuum, we apply one of several trajectory

207 inference methods or dimensionality reduction approaches rather than assessing

208 discrete subpopulations.

209

210 Software availability

211 The method is implemented in Python and can be installed via pip. For the

212 documentation, refer to the package repository at https://github.com/Brodinlab/cellgrid.

214 Acknowledgements

215 P.B and Y.C designed and conceptualized the method. Y.C wrote all the code and

216 implemented the methods. T.L generated the mass cytometry data.

\section{References}

219 Brodin, P. (2019). The biology of the cell - insights from mass cytometry. The FEBS

220 Journal 286, 1514-1522. 
221 Cortes, C., and Vapnik, V. (1995). Support-vector networks. Machine Learning 20, $273-$ 222297.

223 Davis, M.M., and Brodin, P. (2018a). Rebooting Human Immunology. Annu Rev

224 Immunol 36, 1-22.

225 Davis, M.M., and Brodin, P. (2018b). Rebooting Human Immunology. Annual Review of 226 Immunology 36, 843-864.

227 Friedman, J.H. (2001). Greedy function approximation: A gradient boosting machine.

228 The Annals of Statistics 29, 1189-1232.

229 Ho, T. (1998). The random subspace method for constructing decision forests. IEEE

230 Transactions on Pattern Analysis and Machine Intelligence 20, 832-844.

231 Maaten, L. van der (2013). Barnes-Hut-SNE. ArXiv.

232 Macaulay, I.C., Ponting, C.P., and Voet, T. (2017). Single-Cell Multiomics: Multiple

233 Measurements from Single Cells. Trends in Genetics : TIG 33, 155-168.

234 Maecker, H.T., McCoy, J.P., and Nussenblatt, R. (2012). Standardizing

235 immunophenotyping for the Human Immunology Project. Nature Reviews. Immunology 236 12, 191-200.

237 Mair, F., Hartmann, F.J., Mrdjen, D., Tosevski, V., Krieg, C., and Becher, B. (2016). The 238 end of gating? An introduction to automated analysis of high dimensional cytometry 239 data. European Journal of Immunology 46, 34-43.

240 Uhlen, M., Karlsson, M.J., Zhong, W., Tebani, A., Pou, C., Mikes, J., Lakshmikanth, T., 241 Forsström, B., Edfors, F., Odeberg, J., et al. (2019). A genome-wide transcriptomic 242 analysis of protein-coding genes in human blood cells. Sci New York N Y 366.

243 Weber, L.M., and Robinson, M.D. (2016). Comparison of clustering methods for high244 dimensional single-cell flow and mass cytometry data. Cytometry. Part A: The Journal 245 of the International Society for Analytical Cytology 89, 1084-1096.

246 Yu, H.-F., Huang, F.-L., and Lin, C.-J. (2011). Dual coordinate descent methods for 247 logistic regression and maximum entropy models. Machine Learning 85, 41-75. 